Early experience and brain function and structure were investigated in 30 preterm infants (14 controls and 16 experimental) at Children's Hospital, Boston, MA (Als H, Duffy FH, McAulty GB et al. Pediatrics April 2004;113:846-857). The Newborn Individualized Developmental Care and Assessment Program (NIDCAP) was initiated within 72 hours of admission to the intensive care unit and continued to age 2 weeks, corrected for prematurity. The experimental group showed significantly better neurobehavioral functioning at 2 weeks and 9 months assessments. Magnetic resonance diffusion tensor imaging and other tests demonstrated better function and more mature fiber structure for experimental infants compared to controls. Quality of experience before term may accelerate brain development in preterm infants.

\title{
COGNITIVE SIDE EFFECTS OF ANTIEPILEPTIC DRUGS
}

Available studies of the cognitive side effects of traditional and newer antiepileptic drugs (AEDs) are reviewed by researchers at Georgetown University Medical Center, Washington, DC. Relatively few studies in children have meaningful results, most having used global IQ as the primary dependent measure, which may not demonstrate subtle neuropsychological changes. Furthermore, design limitations do not allow firm conclusions about AED cognitive effects. Of older AEDs, phenobarbital (PB) has more negative effects on cognition than valproate (VPA) or carbamazepine (CBZ). PB may decrease IQ, due in part to decreased processing efficiency and poorer attention. The declines in speeded performance are largely reversible, but the long-term effects on academic achievement may be permanent. CBZ does not affect global IQ but it may have a negative effect on memory. Phenytoin (PHT) has a smaller effect on IQ than does PB and its effects on memory are similar to those of CBZ. Effects of VPA on IQ, memory, and academic achievement are dose-dependent but less severe than those of PB, CBZ, and PHT. VPA can be effective in treating disruptive children with explosive temper and mood lability.

Newer AEDs have not been tested using formal neuropsychological methods. Children taking gabapentin (GBP) are at risk for behavioral changes, including hyperactivity, irritability, agitation, and aggression, especially in the mentally retarded. Lamotrigine (LTG) in mentally retarded children with epilepsy is also associated with aggressive behavior, but improved behavior and alertness are reported in children with tuberous sclerosis. Autistic behavior is also improved following LTG, even without seizure control. Levetiracetam (LEV) has conflicting effects on behavior, some studies reporting somnolence and emotional instability, and others finding improvements in cognition, alertness, and behavior. Patients with autism and seizures were also benefited by LEV. Oxcarbazepine (OXC) is associated with somnolence, but no effect on IQ and less nervousness. Topiramate (TPM) may cause emotional lability, fatigue, inattention, and impaired memory, despite improved seizure control. Vigabatrin (VGB) causes visual field constriction in patients with infantile spasms, particularly those with tuberous sclerosis, and is not approved for use in the US. Cases of acute encephalopathy and acute reversible psychosis are reported with VGB. A higher risk of psychotic episodes and obsessive-compulsive disorder is present with zonisamide (ZNS), and symptoms may develop even after prolonged treatment. (Loring DW, Meador KJ. Cognitive side effects of antiepileptic drugs in children. Neurology March (2 of 2) 2004;62:872-877). (Reprints: Dr David W Loring, Department of Neurology, Georgetown University Medical Center, 4000 Reservoir Rd NW, Building D, Suite 207, Washington, DC 20057). 
COMMENT. Studies of the cognitive effects of AEDs in children are few and largely inadequate. Future studies should be controlled and of sufficient duration to evaluate the effects of AEDs on school performance and social adjustment.

\section{NEUROLOGY OF SIGN LANGUAGE}

The neurology of American Sign Language, which originated from French signing about 200 years ago, is reviewed by a pediatric neurologist in the UK. Sign language has its primary site of organization in the dominant hemisphere. Neuroimaging studies have shown the right cerebral hemisphere is also involved in prosodic functions and in the encoding of objects. Studies of congenital and acquired deafness and dysphasia contribute to our knowledge of cerebral localization, especially since the advent of functional MRI and PET. Broca's area of the dominant hemisphere is specialized for sign production, and the nondominant hemisphere is involved with visual-spatial functions and processing of signing, but an interplay between both hemispheres is important in the development of sign language. In childhood epileptic aphasia (Landau-Kleffner syndrome) sign language may provide an alternative means of communication, and it does not impair the acquisition of spoken speech. The importance of sign language in the deaf is shown in the book Seeing Voices (Sacks O, 1991). Cochlear implants may increase in use in the future, but sign language remains the mainstay of communication among the deaf. (Gordon N. The neurology of sign language. Brain Dev 2004;26:146-150). (Respond: Dr Neil Gordon, Huntlywood, 3 Styal Road, Wilmslow SK9 4AE, UK).

COMMENT. Sign language comprising gestures executed in space and dependent on visual spatial orientation might be thought to involve chiefly the non-dominant right cerebral hemisphere. Both hemispheres contribute to sign language but the dominant hemisphere is the primary site of organization.

\section{TOXIC AND VASCULAR DISORDERS}

\section{ACUTE MARIJUANA USE AND CEREBELLAR INFARCTION}

Three adolescent cases of ischemic stroke involving the posterior fossa circulation and cerebellum occurred within days after the illicit use of marijuana and presented over a span of 5 years at St Louis University School of Medicine, MO. Headache, fluctuating consciousness or lethargy, visual disturbance, variable dysphagia/dysarthria, and ataxia were the common presenting manifestations. Cerebellar infarction was confirmed by biopsy (1 case) or necropsy (2 cases). Cerebellar and cerebral edema without brainstem compromise or herniation led to death in the 2 fatal cases. (Geller T, Loftis L, Brink DS. Cerebellar infarction in adolescent males associated with acute marijuana use. Pediatrics April 2004;113:e365-e370). (Reprints: Thomas Geller MD, St Louis University School of Medicine, 1465 S Grand Blvd, St Louis, MO 63104). 